\title{
Problématique de la recherche anthropologique au C.N.R.S.T. (Burkina Faso)
}

Oger Kaboré

\section{OpenEdition}

\section{Journals}

Édition électronique

URL : http://journals.openedition.org/apad/301

DOI : 10.4000/apad.301

ISSN : 1950-6929

Éditeur

LIT Verlag

Édition imprimée

Date de publication : 15 mars 1991

\section{Référence électronique}

Oger Kaboré, «Problématique de la recherche anthropologique au C.N.R.S.T. (Burkina Faso) », Bulletin de l'APAD [En ligne], 1 | 1991, mis en ligne le 23 juin 2006, consulté le 08 septembre 2020. URL : http:// journals.openedition.org/apad/301; DOI : https://doi.org/10.4000/apad.301

Ce document a été généré automatiquement le 8 septembre 2020

Bulletin de l'APAD 


\title{
Problématique de la recherche anthropologique au C.N.R.S.T. (Burkina Faso)
}

\author{
Oger Kaboré
}

1 Dans le cadre de la réunion constitutive de l'association d'Anthropologie du Changement Social et du Développement, je propose ici quelques réflexions sur les problèmes qui se posent à la recherche anthropologique menée au Centre National de la Recherche Scientifique et Technologique (C. N. R. S. T.).

Il faut noter d'abord que la recherche nationale est très jeune au Burkina Faso comme dans la plupart des pays africains où elle a été surtout universitaire avant d'avoir récemment (année 70) un statut autonome. Par conséquent, le manque d'expérience aidant, la recherche se cherche en quelque sorte dans un contexte socio-politique et économique très difficile.

3 La situation de la recherche nationale reste liée à toute la politique de l'État en matière de développement; car c'est lui qui fournit le budget et entend avoir un droit de regard sur la manière dont se mènent les programmes de recherche. Le statut du chercheur demeure donc tributaire de son salaire d'une part, et, d'autre part des moyens qui sont mis à sa disposition pour travailler au développement de son pays.

4 Au Burkina Faso, on note un grand malaise dans le rang des chercheurs lorsque l'on parle de recherche au service du développement. Ce malaise est davantage accentué chez les chercheurs de notre institut de recherche en sciences sociales et humaines (I. R. S. S. H.) dans la mesure où les priorités du développement affichées par l'État ne laissent que peu de concessions aux sciences humaines en général jugées incapables de résoudre les problèmes brûlants de survie (famine, manque d'eau, santé, etc...) Ainsi, dès l'avènement de la révolution, le pouvoir a indexé les intellectuels et surtout les chercheurs (notamment en sciences humaines). On se rappelle cette boutade amère lancée par un journal satirique officiel selon lequel: "des chercheurs qui cherchent on en trouve, mais des chercheurs qui trouvent on en cherche !". Jeu de mots ironique qui 
signifiait à l'époque que la recherche, en particulier en sciences humaines, ne donne pas à manger, ne résout pas les problèmes du pays.

Cette situation a engendré une polémique assez houleuse dans le campus universitaire entre les enseignants des sciences dites exactes ou expérimentales et ceux des sciences humaines, les premiers se prétendant plus utiles au développement que les autres. Tant et si bien que les chercheurs en sciences humaines restent perplexes et mal à l'aise d'autant que, paradoxalement par rapport à leurs ambitions, le budget qui leur est alloué pour la recherche s'est réduit d'année en année comme une peau de chagrin.

Cependant, aujourd'hui, on assiste à une certaine prise de conscience de la part de l'administration de la recherche et aussi des chercheurs des autres disciplines face à la nécessité objective et incontournable d'associer les sciences humaines comme partenaires à part entière dans la conception, la conduite et l'évaluation des programmes de recherche-développement. Ceci évitera aux sciences humaines de jouer, comme elles le font parfois, le rôle de "pompier" là où les projets de développement ont échoué (cf. le colloque national sur le financement et l'utilisation des résultats de la recherche tenu en mai 1990 à Ouagadougou dont l'une des recommandations rejoint les objectifs de la présente association).

7 Ainsi, les instituts du C. N. R. S. T. essaient maintenant d'intégrer les sciences humaines dans leurs programmes de recherche (plan quinquennal ou projets à financements extérieurs). Il est seulement regrettable de constater que les sciences humaines soient réduites à la sociologie ou à la socio-économie et que même ces disciplines ne soient sollicitées souvent que de manière épisodique et instrumentale. Mais cela représente un point positif dans la mesure où le dialogue est amorcé entre la recherche anthropologique (au sens large) et les autres disciplines.

Comme indiqué dans la charte de l'association, il est nécessaire d'élargir le champ du débat pour éviter une utilisation trop restrictive des sciences humaines qui sont plus vastes dans leur ensemble et qui pourraient aider à reposer le problème de l'approche méthodologique du développement de manière plus globale.

Je conclurai en disant que malgré cette situation de ghetto dans laquelle elle se trouve, situation due à des contraintes tant socio-politiques que budgétaires, la recherche anthropologique (parent pauvre) au Burkina peut faire mieux au regard des compétences qui existent dans ce domaine. Le champ d'investigation est très vaste et incite à une recherche qui ne demande qu'à être mieux structurée et organisée. Une stimulation budgétaire et morale engendrerait un dynamisme nouveau chez les chercheurs et produirait ses effets.

Si notre présente association prend corps et fait tâche d'huile, sa démarche pourra impulser un nouveau courant de pensée, une philosophie nouvelle face aux problèmes de développement tels qu'ils se posent dans nos pays africains et singulièrement au Burkina Faso. Elle peut constituer une bouée de sauvetage qui aidera les chercheurs africains à valoriser et faire prévaloir leurs idées dans un dialogue franc avec les développeurs. 